\title{
Performance Analysis of FFH/MFSK Using Division Combining Receiver in GPS System
}

\author{
Li Deng ${ }^{1,2}$, Quanyi Liu ${ }^{1, ~ *, ~ Y u a n h u a ~} \mathrm{He}^{1}$, Qian Chen ${ }^{2}$ \\ ${ }^{1}$ School of Civil Aviation Safety Engineering, Civil Aviation Flight University of China, Guanghan City, China \\ ${ }^{2}$ School of Electronic and Optical Engineering, Nanjing University of Science and Technology, Nanjing, China \\ Email address: \\ 2822993207@qq.com (Quanyi Liu) \\ *Corresponding author \\ To cite this article: \\ Li Deng, Quanyi Liu, Yuanhua He, Qian Chen. Performance Analysis of FFH/MFSK Using Division Combining Receiver in GPS System. \\ Journal of Electrical and Electronic Engineering. Vol. 6, No. 3, 2018, pp. 75-79. doi: 10.11648/j.jeee.20180603.12
}

Received: July 13, 2018; Accepted: July 31, 2018; Published: August 22, 2018

\begin{abstract}
In this paper, a new kind of nonlinear combining receiver of fast frequency hopping/multiple frequency shift keying (FFH/MFSK) modulation in GPS system is proposed. The performance of the receiver is analyzed in the effects of partial band noise jamming over additive white Gaussian noise channel and Ricean fading channel. The diversity of the system is performed using multiple hops per data bit. The non-central F distribution is found in the random variable of the division combining algorithm under the partial-band noise jamming in the additive white Gaussian noise channel. The mathematical results of the bit error ratio of the division combining system are achieved in both fading and non-fading channels. The numerical results are achieved by the simulation environment of the signal transmission while the signal to noise ratio was relatively small and the partial band noise jamming factor is relatively large. It was shown that the GPS system using division combining receiver has better performance under the strong partial band interference than the system without diversity and system with self-normalized combining receiver. This performance gain is because that the interference signal is scattered into several hops and partly diminished by the operation of division algorithm. As its simple structure to be implemented and antiinterference property, the proposed combining algorithm is useful for GPS signal transmission in the strong partial band noise jamming environment.
\end{abstract}

Keywords: FFH, MFSK, Division Combining, Fading Channel

\section{Introduction}

In this paper the transmission characteristic of the proposed FFH/MFSK receiver in the GPS system named division combining receiver $[1,2]$ is analyzed. According to the signal transition environment of GPS channel, the performance is analyzed with multiple hops per data bit under the fading channel $[3,4]$ with partial band noise jamming (PBNJ) and AWGN. The error probability density function (pdf) is derived by the diversity level from one (no diversity) to three. It was shown that when the signal to jamming ratio is relatively low, the proposed MFSK system performances better than the system without diversity and self-normalized combining receiver $[5,6]$ while the diversity level increases. This performance gain is because of the interference signal which is scattered into several hops and partly diminished by the division operations of the system. The combining algorithm is interpreted in the section two and the system performance is analyzed in the following sections. Then the numerical results are shown for comparing the proposed algorithm with the self-normalized combining algorithm which is commonly used in the FFH GPS systems.

\section{System Model}

The structure of the proposed receiver in GPS system is shown in Figure 1. The signal in the GPS antenna is fed to the BPF filter and then disposed by the FFH/MFSK receiver. After that, the signal is combined with division algorithm to shape the decision signal $z$ and finish the demodulation of the GPS system. 


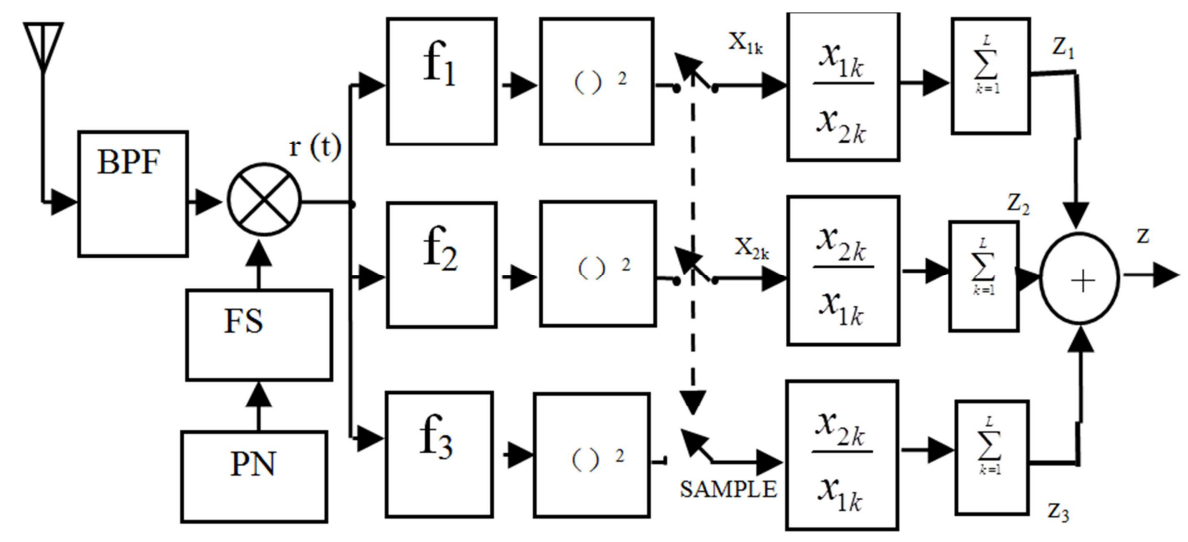

Figure 1. The block diagram of 3-hops division combining receiver.

The GPS signal is assumed to transmits in $\mathrm{L}$ hop/bit FHH/MFSK mode with total bandwidth $\mathrm{W}$, and the baseband frequency $f_{1}, f_{2}$ and $f_{3}\left(f_{1}<f_{2}<f_{3}\right)$ which leads to $M=3$, are transmitted at the rate of $R_{b}=1 / T_{b},\left|f_{3}-f_{1}\right|=B$ which is the cell bandwidth $[7,8]$ equal to hopping rate $R_{h}$. The received signal was passed through the ratio frequency band pass filter and then down converted with the frequency synthesizer (FS) controlled by the PN generator $[9,10]$ coincident with the transmitting end. The dehopped signal $r(t)$ is fed into three channels as shown. The mark and space channels employ square-law envelope detectors whose outputs are sampled every hopping period to produce the quantities $\mathrm{x}_{1 \mathrm{k}}, \mathrm{x}_{2 \mathrm{k}}$ and $\mathrm{x}_{3 \mathrm{k}}$. After that, the signal is brought into the divider whose outputs are $z_{1 k}, z_{2 k}$ and $z_{3 k}$ where $z_{1 k}=x_{1 k} / x_{2 k}, z_{2 k}=x_{2 k} / x_{1 k}$ and $\mathrm{z}_{3 \mathrm{k}}=\mathrm{x}_{3 \mathrm{k}} / \mathrm{x}_{1 \mathrm{k}}$. Because of the presence of the system's thermal noise, the $\mathrm{x}_{1 \mathrm{k}}, \mathrm{x}_{2 \mathrm{k}}$ and $\mathrm{x}_{3 \mathrm{k}}$ will always have positive values(which can not be zeros), so that $z_{1 k}, z_{2 k}$ and $z_{3 k}$ have finite values. Further more, when the power of additive noise is relatively low, the output power is relatively strong, so it looks like that the $E_{b} / N_{0}$ is enlarged by the algorithm. Then $z_{1 k}, z_{2 k}$ and $z_{3 k}$ are summed to form the decision statistics $z_{1}$, $z_{2}$ and $z_{3}$. At last, is $z_{1}, z_{2}$ and $z_{3}$ are compared to make a bit decision. It was assumed that the two adjacent frequency cells of the modulation band are jammed simultaneously with probability $\gamma$, or unjammed simultaneously with probability $1-\gamma$ as in [11]. As a result, when a hop contains very strong interference, the value of $z_{1 k}, z_{2 k}$ and $z_{3 k}$ will be approximately close to the value " 1 ".

The performance of this kind of receiver under the partialband-noise jamming in the AWGN channel is analyzed. The average spectral power density of the PBNJ is $\mathrm{N}_{\mathrm{J}}\left(\mathrm{N}_{\mathrm{J}}=\mathrm{J} / \mathrm{W}\right)$, where $\mathrm{J}$ is the total jamming power. The average spectral power of AWGN is $\mathrm{N}_{0}$. Therefore when a hop is jammed, the power of interference is $\left(\gamma^{-1} \mathrm{~N}_{\mathrm{J}}+\mathrm{N}_{0}\right) \mathrm{B}$ while it is $\mathrm{N}_{0} \mathrm{~B}$ when there is no jamming. It is assumed that the total useful signal power is constant which is equally distributed to L hops where $\mathrm{L}$ is the diversity level.

Since when the average power of the signal and the interference are relatively of great disparity, the abscissa axis of the bit error ratio (BER) curve is chosen from $-10 \mathrm{~dB}$ to $10 \mathrm{~dB}$ to avoid the chance of specific value $z_{1 k}, z_{2 k}$ and $z_{3 k}$ to be a very large number or a quite small number. The performance in the AWGN and the fading channel is discussed below.

\section{Performance Analysis}

A. Performance under the PBNJ in the AWGN channel

Without loss in generality, it was assumed that $f_{1}$ was transmitted in the subsequent analysis. The random variables $\mathrm{x}_{1 \mathrm{k}}, \mathrm{x}_{2 \mathrm{k}}$ and $\mathrm{x}_{3 \mathrm{k}}$ can be represented as follows. [12]

$$
\begin{gathered}
x_{1 k}=\sigma_{k}^{2}\left[\left(\sqrt{\frac{2 S}{\sigma_{k}^{2}}} \cos \theta k+v 1 k\right)^{2}+\left(\sqrt{\frac{2 S}{\sigma_{k}^{2}}} \sin \theta_{k}+v 2 k\right)^{2}\right] \\
x_{2 k}=\sigma_{k}^{2}\left[v_{3 k}^{2}+v_{4 k}^{2}\right] \\
x_{3 k}=\sigma_{k}^{2}\left[v_{5 k}^{2}+v_{6 k}^{2}\right]
\end{gathered}
$$

Where

$$
\sigma_{k}^{2}=\left\{\begin{array}{cl}
N_{0} B, & \text { with probability } 1-\gamma \\
\left(N_{0}+N_{J} / \gamma\right) B, & \text { with probability } \gamma
\end{array}\right.
$$

$\mathrm{S}$ is the signal power and $\theta_{\mathrm{k}}$ is the uniform phase random variables and $v_{i k}, i=1,2,3,4,5,6$ are independent Gaussian random variables with zero means and unit variance.

Thus, $z_{1 \mathrm{k}}$ can be expressed as

$$
z 1 k=\frac{\left(\sqrt{\frac{2 S}{\sigma_{k}^{2}}} \cos \theta k+v 1 k\right)^{2}+\left(\sqrt{\frac{2 S}{\sigma_{k}^{2}}} \sin \theta k+v 2 k\right)^{2}}{\left[v_{3 k}^{2}+v_{4 k}^{2}\right]}
$$

As noticed in paper [1], $\mathrm{z}_{1 \mathrm{k}}$ is a non-central $\mathrm{F}$ distributed random variable with two degrees of freedom with noncentral parameter $2 \mathrm{~S} / \sigma_{\mathrm{k}}{ }^{2}$. According to the structure of the algorithm, the value of $z_{1 k}$ has no relationship with the value of $x_{3 k}$, so $z_{1 k}$ will not be contaminated while the $x_{3 k}$ is interfered by the noise signal. Such the probability density function of $\mathrm{z}_{1 \mathrm{k}}$ can be obtained [13] as below.

$$
f_{z_{1 k}}\left(z_{1 k}\right)=e^{-\frac{\lambda}{2}} \sum_{n=0}^{\infty} \frac{\lambda^{n}(n+1) z_{1 k}^{n}}{2^{n} n !\left(z_{1 k}+1\right)^{n+2}} U\left(z_{1 k}\right)
$$


Where $\lambda=2 S / \sigma_{k}^{2}$ is the non-central parameter of $z_{1 k}, U($.$) is$ the unit step function. And the probability density function of $\mathrm{z}_{2 \mathrm{k}}$ and $\mathrm{z}_{3 \mathrm{k}}$ can also be obtained.

$$
f_{z_{i k}}=e^{-\frac{\lambda}{2}} \sum_{n=0}^{\infty} \frac{\lambda^{n}(n+1)}{2^{n} n !\left(z_{i k}+1\right)^{n+2}} U\left(z_{i k}\right) \quad i=2,3
$$

The probability density function of $z_{1}, z_{2}$ and $z_{3}$ can be get numerically by L-fold convolutions of $f_{z 1 k}, f_{z 2 k}$ and $f_{z 3 k}$. When $\mathrm{L}=2$, the probability density functions are as follows.

$$
f_{z_{i}}\left(z_{i}\right)=\int_{0}^{\infty} f_{z_{i k}}\left(z_{i k}\right) f_{z_{i k}}\left(z_{i}-z_{i k}\right) d z_{i k}
$$

Where $\mathrm{i}=1$ or 2 in equation (8). While $\mathrm{L}$ is above 2 , the probability density functions of $f_{z_{i}}\left(z_{i}\right)$ is as follow.

$$
f_{z_{i}}\left(z_{i}\right)=f\left(z_{i 1}\right) \otimes f\left(z_{i 2}\right) \otimes \cdots \otimes f\left(z_{i L}\right)
$$

Given that $l$ of L hops are jammed, $\operatorname{Pr}(\mathrm{e} \mid l)$ is defined as the conditional probability of error. The probability of bit error is given by

$$
\operatorname{Pr}(e)=\sum_{l=0}^{L}\left(\begin{array}{l}
L \\
l
\end{array}\right) \gamma^{l}(1-\gamma)^{L-l} \operatorname{Pr}(e \mid l)
$$

The function of (10) can be evaluated numerically. Notice that the total signal-to-noise ratio $(\mathrm{SNR})=\left(\alpha^{2}+2 \sigma^{2}\right) /\left(\mathrm{N}_{0} \mathrm{~B}\right)$ and the total signal-to-jamming ratio (SJR) $=\left(\alpha^{2}+2 \sigma^{2}\right) /((\gamma$ ${ }^{1} \mathrm{~N}_{\mathrm{J}}+\mathrm{N}_{0}$ ) B). In the following discussion, it was assumed that the direct component and the diffuse component of the signal have the equal power.

B. Performance under the PBNJ in the fading channel

As in A, it was assumed that $\mathrm{fl}$ was transmitted. Given the signal amplitude $\sqrt{2 S}$, the conditional pdf of $\mathrm{x}_{1 \mathrm{k}}$ is [8]:

$$
f_{x 1 k}(x 1 k \mid S)=\frac{1}{2 \sigma_{k}^{2}} \exp \left(-\frac{x_{1 k}+2 S}{2 \sigma_{k}^{2}}\right) \cdot I_{0}\left(\frac{\sqrt{2 S x_{1 k}}}{\sigma_{k}^{2}}\right)
$$

Where $\alpha^{2}$ is the average power of the direct component of the signal, and $2 \sigma^{2}$ is the average power of the diffuse component of the signal. The total average power of the signal is equal to $\alpha^{2}+2 \sigma^{2}$ and remains constant from hop to hop.

The unconditional pdf [14] of $\mathrm{x}_{1 \mathrm{k}}$ and $\mathrm{x}_{\mathrm{ik}}$ is:

$$
\begin{gathered}
f_{x 1 k}\left(x_{1 k}\right)=\frac{1}{2\left(\sigma_{k}^{2}+2 \sigma^{2}\right)} \exp \left[-\frac{1}{2}\left(\frac{x_{1 k}+2 \alpha^{2}}{\sigma_{k}^{2}+2 \sigma^{2}}\right)\right] \\
\cdot I_{0}\left(\frac{\alpha \sqrt{2 x_{1 k}}}{\sigma_{k}^{2}+2 \sigma^{2}}\right) \\
f x_{i k}\left(x_{i k}\right)=\frac{1}{2 \sigma_{k}^{2}} \exp \left(-\frac{x_{i k}}{2 \sigma_{k}^{2}}\right)
\end{gathered}
$$

In equation (13), parameter $\mathrm{i}$ indicates the number of the hops $\mathrm{L}, \mathrm{L}=2,3, \ldots \mathrm{n}$. Then the pdf of $\mathrm{z}_{1 \mathrm{k}}$ and $\mathrm{zi}_{\mathrm{k}}$ can be calculated as below:

$$
\begin{aligned}
f z_{1 k}\left(z_{1 k}\right) & =\int_{0}^{\infty} f x_{1 k}\left(z_{1 k}-x_{2 k}\right) f x_{2 k}\left(\frac{1}{x_{2 k}}\right) \frac{1}{x_{2 k}^{2}} d x_{2 k} \\
f z_{i k}\left(z_{i k}\right) & =\int_{0}^{\infty} f x_{i k}\left(z_{i k}-x_{1 k}\right) f x_{1 k}\left(\frac{1}{x_{1 k}}\right) \frac{1}{x_{1 k}^{2}} d x_{1 k}
\end{aligned}
$$

After this the close-form of the probability density functions of $\mathrm{z}_{1 \mathrm{k}}$ and $\mathrm{z}_{\mathrm{ik}}$ are:

$$
\begin{gathered}
f_{z 1 k}(z 1 k)=\frac{\alpha^{2} \sigma_{k}^{4} z_{1 k}+\left(\sigma_{k}^{4}+2 \sigma^{2} \sigma_{k}^{2}\right)\left(\sigma_{k}^{2}+2 \sigma^{2}+\sigma_{k}^{2} z_{1 k}\right)}{\left(\sigma_{k}^{2}+2 \sigma^{2}+\sigma_{k}^{2} z_{1 k}\right)^{3}} \\
\cdot \exp \left(-\frac{\alpha^{2}}{\sigma_{k}^{2}+2 \sigma^{2}+z_{1 k}}\right) \\
f_{z i k}(z i k)=\frac{\alpha^{2} \sigma_{k}^{4}+\left(\sigma_{k}^{4}+2 \sigma^{2} \sigma_{k}^{2}\right)\left(\sigma_{k}^{2}+2 \sigma^{2} z_{i k}+\sigma_{k}^{2} z_{i k}\right)}{\left(\sigma_{k}^{2}+2 \sigma^{2} z_{i k}+\sigma_{k}^{2} z_{i k}\right)^{3}} \\
\cdot \exp \left(-\frac{\alpha^{2} z_{i k}}{\sigma_{k}^{2}+2 \sigma^{2} z_{i k}+\sigma_{k}^{2} z_{i k}}\right)
\end{gathered}
$$

The probability of bit error in the fading channel can be get through equation (9) and (10).

\section{Numerical Results}

The following figure 2 and figure 3 show the bit errors of the FFH/MFSK system in the AWGN channel with the PBNJ interference. Figure 2 shows the performance of the GPS system with $\mathrm{E}_{\mathrm{b}} / \mathrm{N}_{0}=13.35 \mathrm{~dB}$. One can see that in the presence of strong PBNJ $\left(E_{b} / N_{J}\right.$ from -10 to $\left.3 \mathrm{~dB}\right)$, the diversity receiver performances better than the receiver without the diversity when the jamming factor $\gamma$ is 0.5 and 0.1 . The performance becomes much better when $\gamma$ decreases.

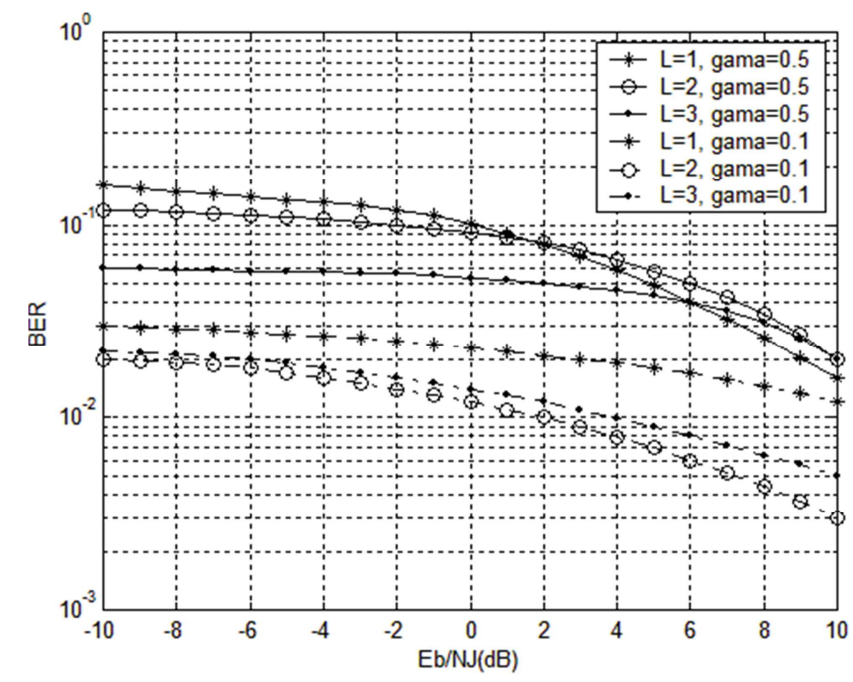

Figure 2. BER under PBNJ in the AWGN channel with different gama. 
Figure 3 shows the comparison between division combining and self-normalized combining receiver in the AWGN channel with the jamming factor $\gamma=0.1$ at different PBNJ ratio. The results show the proposed receiver has better performance at the same diversity level with the increasing of $\mathrm{E}_{\mathrm{b}} / \mathrm{N}_{\mathrm{J}}$. When the diversity level increase, both two kinds of receiver have better BER performance.

Figure 4 and figure 5 show the system performance under the PBNJ in frequency non-selective slowly Ricean fading channel with different partial band noise jamming factor $\gamma$ while the $\mathrm{E}_{\mathrm{b}} / \mathrm{N}_{\mathrm{J}}$ ratio is $0 \mathrm{~dB}$.

It can be observed that in the Ricean fading channel, the bit error performance becomes insensitive to the $\mathrm{E}_{\mathrm{b}} / \mathrm{N}_{0}$ ratio when the jamming factor $\gamma$ gets larger. However, in the presence of strong PBNJ, the division combining receiver has better performance than the receiver without the diversity and the self-normalized combining receiver. While the jamming factor $\gamma$ is relatively small as in figure 5 , the diversity system gets better algorithms gain with the increasing of $\mathrm{E}_{\mathrm{b}} / \mathrm{N}_{0}$ ratio.

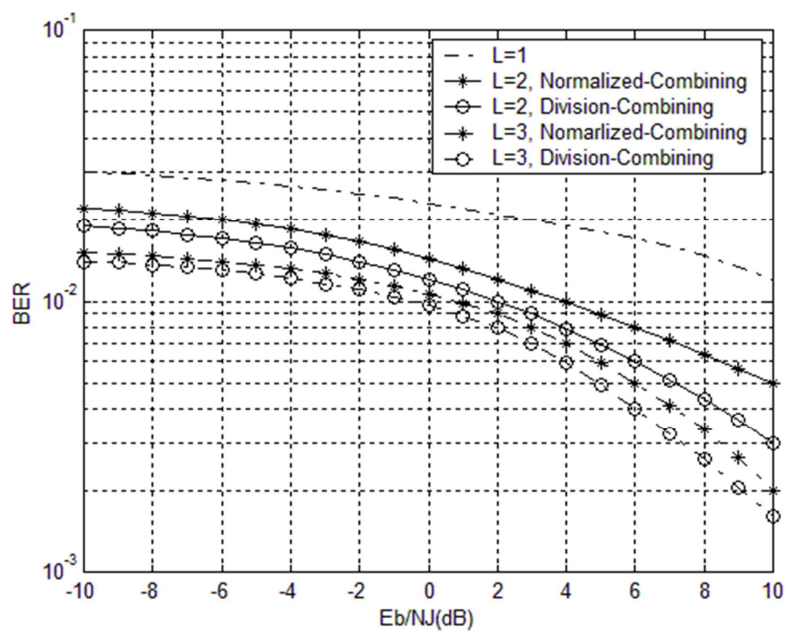

Figure 3. Comparison of BER under PBNJ in the AWGN channel.

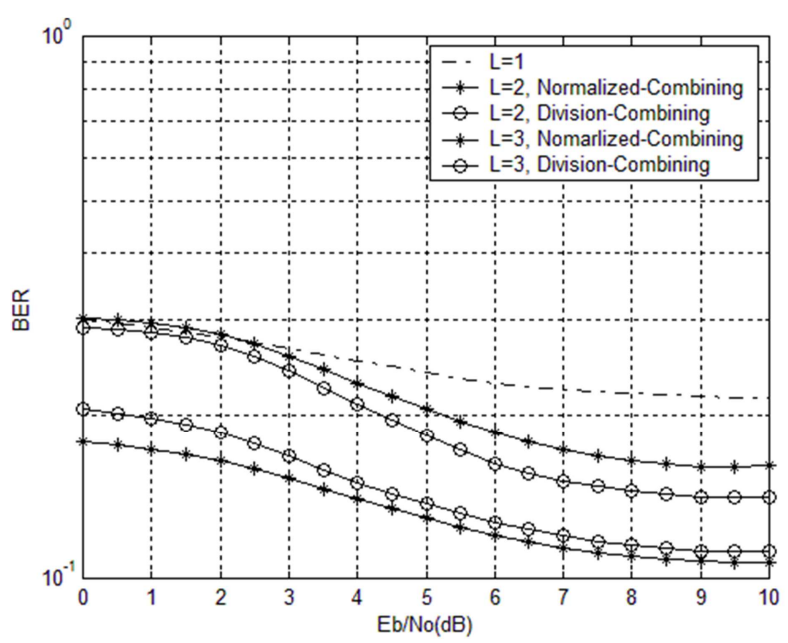

Figure 4. Comparison of BER in the fading channel with gamma=0.5

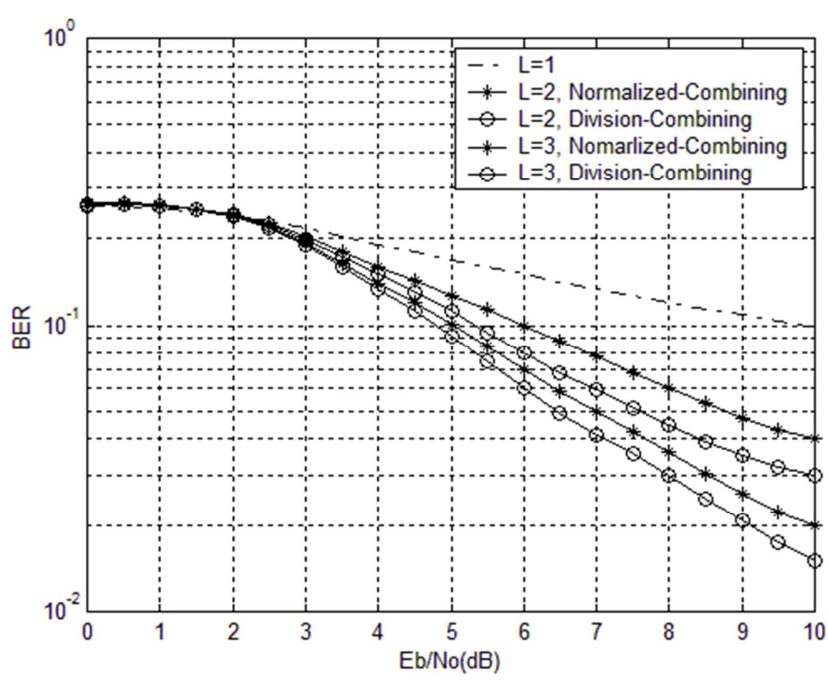

Figure 5. Comparison of BER in the fading channel with gamma=0.1.

\section{Conclusion}

In this paper the performance of division combining receiver of FFH/MFSK in GPS system is analyzed in the presence of strong interference $\left(\mathrm{E}_{\mathrm{b}} / \mathrm{N}_{\mathrm{J}}<10 \mathrm{~dB}\right)$ while the hop $\mathrm{L}=3$. The results show that the diversity receiver has better performance than the system without diversity and self-normalized combining receiver [15] in the AWGN channel and Ricean fading channel under partial-bandnoise jamming. It was shown the proposed combining algorithm performances better than the self-normalized combining method at the lower $\mathrm{E}_{\mathrm{b}} / \mathrm{N}_{\mathrm{J}}$ ratio $(<10 \mathrm{~dB})$ with the same $E_{b} / N_{0}$ ratio while the $L$ increases. The division combining receiver has simpler structure than the selfnormalized combining receiver and has easy way to be implemented. It will be useful for GPS signal transmission in the strong partial-band-noise jamming environment. However, when the dividing hops increase the computation complexity of the algorithm will also increase a lot. Meanwhile all of the random variety $\mathrm{z}_{1}, \mathrm{z}_{2}, \ldots, \mathrm{z}_{\mathrm{L}}$ will depends on the quantity of $\mathrm{x}_{1 \mathrm{k}}$ which may be randomly interfered by the partial band jamming. So without loss of generality, the random $i$ of the $x_{i k}$ can be chosen for dispatching the interference to every hop of the random variety in the further research works.

\section{Acknowledgements}

The work described in this paper were supported by Key Program of National Natural Science Foundation of China (No.: U1633203, No.: U1733126), the major project funded by Civil Aviation Administration of China (MHRD20160103) and the Scientific Research Fund Project of CAFUC (J2016-41, J2018-36). The authors deeply appreciate the supports. 


\section{References}

[1] L. Deng, K. Xu, Q. Wang and J. Sun, "Performance analysis of FFH/BFSK using division combining receiver in GPS system," 2016 12th World Congress on Intelligent Control and Automation (WCICA), Guilin, 2016, pp. 5-8.

[2] H. Mezaoui, A. M. Hamza and P. T. Jayachandran, "Dynamic analysis of the polar ionosphere using the GPS signal: Toward an optimization of the cutoff scale," in Radio Science, vol. 52, no. 2, pp. 271-281, Feb. 2017.

[3] Wang C, Walker R A, Moody M P. A GPS Signal Transmission Model for Improved Signal Antenna Attitude Determination. 2005

[4] R. He, B. Ai, G. L. Stüber and Z. Zhong, "Mobility ModelBased Non-Stationary Mobile-to-Mobile Channel Modeling," in IEEE Transactions on Wireless Communications, vol. 17, no. 7, pp. 4388-4400, July 2018.

[5] Y. He et al., "Performance analysis of a subset-based coherent FFH system with spatial modulation in Rayleigh fading channels with multitone jamming," in IET Communications, vol. 10, no. 16, pp. 2199-2205, 1132016.

[6] M. D. Theodoss and R. C. Robertson, "Performance of the FFH/BFSK self-normalized receiver with convolutional coding and soft decision decoding over Rician fading channels with partial-band noise interference," Military Communications Conference, 1996. MILCOM '96, Conference Proceedings, IEEE, McLean, VA, 1996, pp. 436441 vol. 2.

[7] Mattbew S Gast.802.11 Wireless Networks:The Definitive Guide. 2005.

[8] E. Schmidt, Z. A. Ruble, D. Akopian and D. J. Pack, "A reduced complexity cross-correlation interference mitigation technique on a real-time software-defined radio GPS L1 receiver," 2018 IEEE/ION Position, Location and Navigation Symposium (PLANS), Monterey, CA, 2018, pp. 931-939.

[9] A. Tabatabaei, M. R. Mosavi and H. S. Shahhoseini, "MP Mitigation in Urban Canyons using GPS-combinedGLONASS Weighted Vectorized Receiver," in IET Signal Processing, vol. 11, no. 4, pp. 446-451, 62017.

[10] Landry, R. J, Calmettes, V., Bousquet, M. Impact of interference on a generic GPS receiver and assessment of mitigation techniques. Spread Spectrum Techniques and Applications. 1998

[11] J. S. Lee, L. E. Miller and Y. K. Kim, "Probability of error analysis of a BFSK frequency-hopping system with diversity under partial-band jamming interference-Part II :Performance of a square law nonlinear combining -soft decision receivers," IEEE Trans. Commun., vol. COM-32, no. 12, pp. 1243-1250, December 1984.

[12] D. Whalen, Detection of Signals in Noise. New York: Academic, 1971.

[13] M. Abramowitz and I. A. Stegun, Eds., Handbook of Mathematical Functions. Washington, DC: Gbv. Print. Off., 1964.

[14] N. Milosevic, Z. Nikolic, and D. Paunovic, "Performance analysis of FFH/FSK system using diversity," in Proc. 5th Int. Conf. Telecommunications in Modern Satellite, Cable and Broadcasting Service (TELSIKS'01), Sept. 2001, pp. 263-266.

[15] Ye-Shun Shen and Szu-Lin Su, "Performance analysis of an FFH/BFSK receiver with self-normalizing combining in a fading channel under independent multitone interference," Global Telecommunications Conference, 2002. GLOBECOM '02. IEEE, 2002, pp. 1319-1323 vol.2. 